\title{
Mixed lineage kinase domain-like pseudokinase mediated necroptosis aggravates periodontitis progression
}

Yanan Yang ( $\nabla$ yyanan56@126.com )

Tongji University https://orcid.org/0000-0003-4981-3582

Lingxia Wang

Chinese Academy of Sciences

Haibing Zhang

Chinese Academy of Sciences

Lijun Luo

Tongji University https://orcid.org/0000-0001-7137-1739

\section{Research Article}

Keywords: necroptosis, periodontitis, cell death, alveolar bone loss, inflammation

Posted Date: April 28th, 2021

DOI: https://doi.org/10.21203/rs.3.rs-468306/v1

License: (a) (i) This work is licensed under a Creative Commons Attribution 4.0 International License.

Read Full License

Version of Record: A version of this preprint was published at Journal of Molecular Medicine on October 13th, 2021. See the published version at https://doi.org/10.1007/s00109-021-02126-7. 


\section{Abstract}

Necroptosis is a form of cell death that is reportedly involved in the pathogenesis of periodontitis. However, the role of Mlk/involved necroptosis remains unclear. Herein, we aim to explore the role of MLKL-mediated necroptosis in periodontitis in vitro and in vivo. Expression of RIPK3, MLKL, and phosphorylated MLKL is observed in gingival tissues obtained from healthy subjects or patients with periodontitis. Viability of Porphyromonas gingivalis lipopolysaccharide (LPS-Pg)-treated cells was detected. In wild type or MIk/ deficiency mice with ligature-induced periodontitis, alveolar bone loss and osteoclast activation were assessed. mRNA levels of inflammatory cytokines in bone marrow-derived macrophages were tested by qRT-PCR. Increased expression of RIPK3, MLKL, and phosphorylated MLKL is observed in gingival tissues obtained from patients with periodontitis. Porphyromonas gingivalis lipopolysaccharide (LPS- $P g$ )-treated cells developed necroptosis after caspase inhibition and negatively regulated the NF-KB signaling pathway. In mice with ligature-induced periodontitis, MIk/ deficiency reduced alveolar bone loss and weakened osteoclast activation. Furthermore, genetic ablation of Mlk/ in LPS-Pg-treated bone marrow-derived macrophages increased the mRNA levels of tumor necrosis factor-a, interleukin (II)-1 $\beta$, II-6, cyclooxygenase 2, matrix metalloproteinase 9 , and receptor activator of nuclear factor kappa-B ligand. Our data indicated that MLKL-mediated necroptosis aggravates the development of periodontitis in a Mlk/deficient mouse. And this will provide a new sight for the understanding of etiology and therapies of periodontitis.

\section{Key Messages}

- MLKL expression was upregulated in human gingival tissue.

- Mlk/ deficiency affected the progression of periodontitis.

- Necroptosis played a major role in mice periodontitis model.

- Knockout of $M / k /$ had a significant effect on inflammatory responses.

\section{Introduction}

Periodontitis, a chronic inflammatory disease induced by a pathogenic periodontal microbiome, leads to destruction of the periodontium. The prevalence of periodontitis is as high as $45-50 \%$, the severe periodontitis affects $11.2 \%$ of the world's population [1]. It is one of the major oral diseases and affects about half of the adult population worldside [2]. Moreover, periodontitis is a risk factor for many systemic conditions, including cardiovascular disease, lung disease, preterm labor, and diabetes mellitus [3, 4]. Periodontal homeostasis is achieved by intricate regulatory mechanisms functioning in concert [5]. For example, cell proliferation and cell death are two essential processes in maintaining homeostasis in metazoans, and disturbances in these processes may lead to disease development.

Currently, apoptosis, necroptosis, autophagy and pyroptosis are recognized forms of cell death [6]. Recent studies have demonstrated that a failure to regulate cell death may be involved in the pathogenesis of inflammatory diseases [7-9]. During necroptosis, immunostimulants are released inside the cell, and 
therefore, inflammation is an important pathological feature of necroptosis [10-12]. Although some studies have shown that the pathogenesis of periodontitis is related to autophagy or apoptosis, little is known about the link between necroptosis and periodontitis [13-15].

The pathogenesis of various inflammatory diseases, such as inflammatory bowel disease is closely linked to necroptosis [16]. At the molecular level, receptor-interacting protein kinase 1 (RIPK1) plays a crucial role in mediating caspase-independent necroptosis, which is suppressed by a small-molecule inhibitor of RIPK1, necrostatin-1 (Nec-1) [17]. Experimentally, caspase-independent necroptosis can be initiated by a pan-caspase inhibitor, such as zVAD-fluoromethyl ketone (zVAD) [18]. Receptor interacting protein kinase 3 (RIPK3) is a key driver of necroptosis and is induced by cell death triggers, such as cytokines (for example, tumor necrosis factor receptor 1 ) [19, 20], toll-like receptors (TLRs) [21, 22], intracellular RNA and DNA sensors [23, 24]. Mixed lineage kinase domain-like pseudokinase (MLKL) is an effector of necroptosis and is downstream of RIPK3 $[16,25]$. Phosphorylation of MLKL (p-MLKL) by RIPK3 induces its oligomerization. The oligomer is then transferred to the plasma membrane where it interacts with phosphatidylinositol, penetrates the cell membrane penetration and causes cell death [17]. At present, Mlkl-deficient mice are considered to be the best tool for investigating necroptosis [26].

Recently, several reports have shown that necroptosis is involved in the pathogenesis of periodontitis [27-29]. In this study, we explored MLKL-mediated necroptosis both in vitro and in vivo. Our findings could provide valuable insights into the role of necroptosis in the pathogenesis of periodontitis and add to our current understanding of periodontitis.

\section{Materials And Methods}

\section{Ethics statement}

This study, including the method of obtaining consent, was approved by the Clinical Ethics Committee of at the School of Stomatology, Tongji University, in accordance with government-issued guidelines and institution policies (Approval number 2018012). Written informed consent was obtained from all the participants involved in this study. All subjects were over 18 years of age, were assessed for the presence/history of periodontal disease. Smokers, pregnant women, and nursing women were excluded from the study. Probing depth, clinical attachment loss, bleeding on probing, and physiological bone loss were assessed. The periodontitis group consisted of subjects with severe periodontitis, but without other serious systemic conditions. The healthy group consisted of volunteers in general good health who showed no signs of clinical attachment loss, no physiological bone loss with probe depth $\leq 3 \mathrm{~mm}$, instances of bleeding on probing were $<10 \%$, and there was no visible gingival inflammation [30]. A gingival biopsy was collected from each subject (4 per group).

\section{Mice}

Specific pathogen-free male mice were used in this study. Mlkl-deficient $\left(\mathrm{Mlkl}^{\prime-}\right)$ mice shared a common genetic C57BL/6 background with wild-type (WT) mice. Animal experiments were conducted in 
accordance with the guidelines of the Clinical Ethics Committee at the School of Stomatology, Tongji University (Approval number 2018011).

\section{Cell culture}

WT and $\mathrm{MlkF}^{-1-}$ mice, aged 6-8 weeks, were sacrificed by cervical dislocation. The mice were sanitized by immersing them in $75 \%$ alcohol for $2 \mathrm{~min}$. To harvest and culture bone marrow-derived macrophages (BMDMs), the intact femur and tibia were removed and placed in phosphate-buffered saline solution. Both ends of the bone were then removed and the bone marrow cavity was rinsed with Dulbecco's modified Eagle's medium (DMEM) until the cavity turned white. Cells were harvested at room temperature, centrifuged, and resuspended in DMEM supplemented with $10 \%$ fetal bovine serum, $100 \mathrm{U} / \mathrm{mL}$ penicillin, $100 \mu \mathrm{g} / \mathrm{mL}$ streptomycin, and $20 \mathrm{ng} / \mathrm{mL}$ macrophage colony-stimulating factor (PeproTech, Rocky Hill, NJ, USA). L929 cells were cultured in DMEM supplemented with $10 \%$ fetal bovine serum, $100 \mathrm{U} / \mathrm{mL}$ penicillin, and $100 \mu \mathrm{g} / \mathrm{mL}$ streptomycin.

\section{Western blots}

Gingival tissues of healthy individuals and patients with periodontitis were obtained. Frozen gingival tissues were homogenized rapidly in liquid nitrogen and lysed on ice in a lysis buffer comprised of 50 $\mathrm{mM}$ Tris [pH 7.4], $150 \mathrm{mM} \mathrm{NaCl}, 1 \%$ Triton $\mathrm{X}-100,1 \%$ sodium deoxycholate, $0.1 \%$ sodium dodecyl sulf, sodium orthovanadate, sodium fluoride, EDTA, and leupeptin, supplemented with $1 \%$ protease inhibitor cocktail (Bimake, Houston, TX, USA). After $30 \mathrm{~min}$, the lysates were centrifuged at $12,000 \mathrm{~g}$ for $15 \mathrm{~min}$ at $4^{\circ} \mathrm{C}$. The protein concentration was determined using the bicinchoninic acid method (Beyotime, Shanghai, China). Twenty micrograms of total protein were separated on an $8 \%$ sodium dodecyl sulfate polyacrylamide gel via electrophoresis, then transferred onto polyvinylidene difluoride membranes (EMD Millipore, Billerica, MA, USA). After complete protein transfer, the membranes were blocked with $5 \%$ milk powder solution for $2 \mathrm{~h}$ at room temperature and incubated overnight at $4^{\circ} \mathrm{C}$ with the following rabbit monoclonal anti-RIPK3 (Abcam, Shanghai, China), anti-MLKL (Proteintech, Rosemont, IL, USA), antiphospho-MLKL (p-MLKL) (Abcam), or anti-GAPDH (Cell Signaling Technology, Danvers, MA, USA). Immunodetection was performed using the Odyssey CLx (LI-COR, Lincoln, NE, USA), and the blots were quantified using ImageJ. Treated L929 cells similarly subjected to western blotting.

\section{Cell viability assay}

Cells were seeded into 96-well plates and treated with $1 \mu \mathrm{g} / \mathrm{mL}$ Porphyromonas gingivalis lipopolysaccharide (LPS-Pg, InvivoGen, San Diego, CA, USA), $20 \mu \mathrm{M}$ of the pan-caspase inhibitor, zVAD (Promega, Madison, WI, USA) or $30 \mu \mathrm{M} \mathrm{Nec-1}$ (Cambridge Bioscience, Cambridge, UK) for $24 \mathrm{~h}$. Cell survival was determined using the CellTiter-Glo luminescent cell viability assay (Promega). Luminescence was read using an Infinite M200 microplate reader (Tecan, Zürich, Switzerland).

\section{RNA extraction, reverse transcription and the quantitative real-time polymerase chain reaction (qRT- PCR)}


Total RNA was extracted using TRIzol (Life Technologies, Carlsbad, CA, USA). The quantity of RNA was determined using a Nanodrop ND-1000 spectrophotometer (Thermo Fisher Scientific, Waltham MA, USA). One microgram of RNA was used for reverse transcription using the RT reagent kit (Takara, Kusatsu, Japan). The qRT-PCR was performed using SYBR Green PCR Master Mix in a LightCycler 480® (Roche, Basel, Switzerland). Fold change in mRNA levels was calculated by the Eq. $2^{-\Delta \Delta C t}$ method [31]. Primer sequences are shown in the Table.

\section{Ligature-induced experimental periodontitis}

The experimental periodontitis model was induced in WT and $\mathrm{MlkT}^{-/-}$mice at eight weeks of age (ten per group). A 5 - 0 silk ligature was tied around the maxillary right second molar, while the contralateral unligated tooth served as the baseline control. Alveolar bone loss (ABL) was analyzed 7 days after ligature placement. For this, the distance between the cementoenamel junction and the alveolar bone crest (CEJ-ABC distance) was measured on both the buccal and palatal sides. Further, the maxillae of the mice were subjected to micro-computed tomography (CT) and histological analyses. Mice in which the ligatures were lost were excluded from the analyses.

\section{Bone loss measurements}

For micro-CT analysis, the maxillae were fixed in $4 \%$ paraformaldehyde until analysis. To calculate the $A B L$, the $C E J-A B C$ distance on the control tooth was subtracted from that on the ligated tooth.

\section{Histological analyse}

For histological analyses, the maxillae were fixed in $4 \%$ paraformaldehyde, decalcified in $10 \%$ EDTA solution for 3 weeks, and then embedded in paraffin. Sections were stained with hematoxylin and eosin and tartrate-resistant acid phosphatase (TRAP). TRAP-positive multinucleated ( $>3$ nuclei) cells were counted as osteoclasts.

\section{Statistical analyse}

Statistical significance between the groups was determined using the two-tailed Student's t-test or Oneway ANOVA. All analyses were performed using Prism 7.0 software (GraphPad, La Jolla, CA, USA). $P<$ 0.05 was considered significant.

\section{Results}

\section{Necroptosis is involved in periodontitis}

The upregulation of proteins belonging to the necroptosis machinery, such as RIPK3 and, more specifically, MLKL and p-MLKL, is a strong indication of necroptosis [32]. We evaluated protein and transcript levels of RIPK3, MLKL, and p-MLKL in gingival tissues from healthy subjects and patients with periodontitis using western blots and qRT-PCR. In periodontitis patients, RIPK3, MLKL, and p-MLKL 
protein levels increased dramatically compared to those in healthy patients (Fig. 1A, B). Similarly, Ripk3 and MIk/mRNA levels also increased in patients with periodontitis compared to those in healthy subjects (Fig. 1C). Collectively, these results suggest that necroptosis is activated in periodontitis.

\section{LPS-Pg and zVAD treatment of cells activates necroptosis and negatively regulates the NF-KB signaling pathway}

Necroptosis can be activated via distinct pathways, including TLR4-mediated signaling [33]. P. gingivalis is a key pathogen that causes periodontitis, and LPS derived from $P$. gingivalis is known to stimulate TLR4 [34]. In this study, BMDMs from WT mice, and L929 cells, were treated with the pan-caspase inhibitor, zVAD in conjunction with LPS-Pg or Nec-1 (a RIPK1 inhibitor) for $24 \mathrm{~h}$. Interestingly, exposure to LPS-Pg alone was not sufficient to induce necroptosis in either BMDMs or L929 cells. Cells co-incubated with zVAD and LPS-Pg exhibited necroptosis, while incubation with Nec-1 reversed this effect, indicating that LPS-Pg treated cells exhibit caspase-independent necroptosis (Fig. 2A, B). The expression of proteins involved in the mitogen-activated protein kinase/c-jun N-terminal kinase and NF-kB signaling pathways were detected in L929 cells. Thus, the survival rates of L929 cells treated with the corresponding inhibitors of these pathways were assessed (Fig. 2C, D). p65 and p-p65 protein levels decreased in cells treated with LPS-Pg and ZVAD, leading to the activation of necroptosis. On the other hand, 665 inhibitor treatment further sensitized L929 cells to LPS-Pg and ZVAD stimulation, leading to a more profound activation of necroptosis. This suggests that activation of the NF-KB signaling pathway prevents LPS-Pgmediated necroptosis.

\section{MIk/ deficiency mitigates alveolar bone loss in mice with ligature-induced periodontitis}

To validate the role of MLKL-mediated necroptosis in the pathogenesis of periodontitis, an experimental model of periodontitis was established in WT and $\mathrm{MlkJ}^{-1-}$ mice. Micro-CT scans of ligated teeth revealed significantly more alveolar bone resorption in WT compared to $\mathrm{Mlkl}^{-1-}$ mice (Fig. 3A). Although the CEJ$A B C$ distances were different between the two groups (Fig. 3B), the buccal side, especially sites corresponding to cusps, presented milder alveolar bone loss in Mlk ${ }^{1-}$ compared with WT mice (Fig. 3C). These results indicate that $M / k /$ deficiency protects against alveolar bone loss.

\section{MIk/ deficiency weakens the osteoclast activation in ligature-induced periodontitis mice}

TRAP staining demonstrated that the number of osteoclasts increased in the ligation model compared with that in the control (Fig. 4A). However, the number of osteoclasts decreased in $\mathrm{MlkF}^{-1-}$ compared with WT mice (Fig. 4B). Hematoxylin and eosin staining revealed an infiltration of inflammatory cells, a loss of connective tissue attachment, and a resorption of the alveolar bone in gingival tissue. Inflammation and alveolar bone loss were observed in both WT and $\mathrm{MlkJ}^{-1-}$ mice (Fig. 4C). These results suggest that MlkI deficiency weakens osteoclast activation in mice with ligature-induced periodontitis.

\section{LPS-Pg induces differential expression of inflammatory cytokine- and osteoclast-related genes in BMDMs from WT and $\mathrm{Mlkr}^{--}$mice}


To determine the role of $M l k l$ in regulating the expression of cytokine- and osteoclast-related genes, BMDMs from WT and $\mathrm{Mlk}^{-1-}$ mice were cultured in vitro and stimulated with LPS-Pg for different times. Transcript levels of the inflammatory cytokine genes, tumor necrosis factor-a, interleukin (II)-1 $\beta$, cyclooxygenase 2, and II-6, (Fig. 5A), and of the osteoclast-related genes, matrix metalloproteinase 9 and receptor activator of nuclear factor kappa-B ligand, were measured by qRT-PCR. A significant difference was observed in expression of inflammatory cytokine and osteoclast-related genes between BMDMs from WT and $\mathrm{MlkT}^{-1-}$ mice (Fig. 5B). In the absence of LPS- $P g$ treatment, a difference in expression of the aforementioned genes was not found. Although expression of the cytokine genes in BMDMs from WT

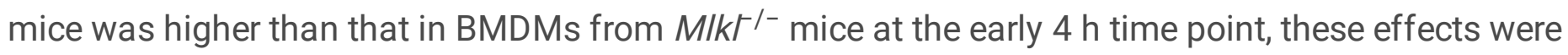
reversed later. Similar to the expression of cytokine genes, osteoclast-related genes showed a decrease in expression in BMDMs from $\mathrm{MlkF}^{-1-}$ compared to WT mice. Altogether, our data demonstrate that MLKLmediated necroptosis is required for LPS-induced expression of cytokine- and osteoclast-related genes.

\section{Discussion}

Periodontitis is an inflammatory response of the host's immune system against periodontal pathogenic bacteria in an attempt to clear the bacterial invasion; however, this occurs at the cost of a loss of periodontal tissue. In this context, cell proliferation and cell death are two essential elements maintaining homeostasis in periodontal tissue [6]. Recently, necroptosis was identified as a form of cell death that occurs during infection and sterile inflammation. Increased expression of RIPK3 and MLKL is an indicator of necroptosis [26]. Both RIPK3- and MLKL-dependent necroptosis have been reported to be involved in systemic inflammatory conditions such as accelerating tissue injury in acute pancreatitis (He et al. 2009). Ke et al. reported that $P$. gingivalis may cause necroptosis during the development of periodontitis [28]. Phosphorylated (p)-MLKL is considered a specific marker of necroptosis [35]. When we analyzed gingival biopsies from healthy subjects and patients with periodontitis, it was found that RIPK3, MLKL, and pMLKL protein levels were significantly higher in the latter. These results corroborate the finding that necroptosis occurs in periodontitis. However, whether the upregulation of RIPK3 and/or MLKL serves as a primary causal factor or is a secondary consequence of persistent inflammation remains unclear and needs to be further studied [8].

Kondylis et al. reported that the IKK/NF-KB signaling pathway inhibits RIPK1 activity-dependent cell death, thus protecting the host against a homeostatic imbalance and inflammation in the intestine [36]. Activation of the NF-KB signaling pathway promotes cell survival, cell proliferation, and the production of pro-inflammatory cytokines [17]. This is confirmed by our finding that LPS- $P g$-treated cells, incubated with a caspase inhibitor, developed necroptosis and exhibited negative regulation of the NF-KB signaling pathway. Necroptosis triggers the release of pro-inflammatory cytokines, including tumor necrosis factor and interferon, which are potent inducers of necroptosis, further prolonging inflammation [8]. MLKL is less pleiotropic than RIPK1 and RIPK3. Thus, $\mathrm{Mlk/}^{-1-}$ mice are preferred for investigating necroptosis in the experimental periodontitis model used in the present study [26]. Differential expression of inflammatory cytokine- and osteoclast-related genes was observed in WT and $\mathrm{MlkI}^{-1-}$ mice. ABL and 
osteoclast activation were suppressed in $\mathrm{MlkI}^{- \text {- }}$ compared with WT mice, suggesting that Mlk/ deficiency mitigated inflammation.

In conclusion, MLKL mediates necroptosis in inflamed periodontal tissues. Previous studies investigating the relationship between periodontitis and necroptosis have focused on cellular or WT animal models [27, 28]. Our study is the first to validate the fact that necroptosis aggravates the development of periodontitis in a Mlk-deficient mouse model. However, how necroptosis interacts with other proinflammatory signaling pathways in the pathogenesis of periodontitis needs to be investigated further. Identifying necroptosis inhibitors may lead to the development of new immunotherapies for periodontitis.

\section{Declarations}

\section{Acknowledgments and Funding Information}

This study was supported by the National Natural Science Foundation of China (No. 82071123, China) and the research fund of Shanghai Municipal Commission of Health and Family Planning (project 201740232 and project 20174Y0132).

Funding: This study was supported by the National Natural Science Foundation of China (No. 82071123, China) and the research fund of Shanghai Municipal Commission of Health and Family Planning (project 201740232 and project 20174Y0132).

Conflicts of interest: There are no potential conflicts of interest with this article.

Availability of data and material: All data generated or analyzed during this study are included in this published article.

Code availability: Not applicable.

Authors' contributions: Yanan Yang performed all experiments, interpreted the results and drafted the manuscript. Lingxia Wang was involved in samples acquisition. Lijun Luo and Haibing Zhang contributed to conception and design of the study. Lijun Luo conceived the idea for the project, supervised the project, critically revised the manuscript and have given final approval of the version to be published.

Ethics approval: This study, including the method of obtaining consent, was approved by the Clinical Ethics Committee of at the School of Stomatology, Tongji University, in accordance with governmentissued guidelines and institution policies (Approval number 2018012).

Consent to participate: Not applicable.

Consent for publication: Not applicable.

\section{References}


1. Kassebaum NJ, Bernabé E, Dahiya M, Bhandari B, Murray CJL, Marcenes W (2014) Global burden of severe periodontitis in 1990-2010: a systematic review and meta-regression. J Dent Res 93: 10451053. DOI 10.1177/0022034514552491

2. Holde GE, Oscarson N, Trovik TA, Tillberg A, Jonsson B (2017) Periodontitis Prevalence and Severity in Adults: A Cross-Sectional Study in Norwegian Circumpolar Communities. Journal of periodontology 88: 1012-1022. DOI 10.1902/jop.2017.170164

3. Hayashi C, Gudino CV, Gibson FC, 3rd, Genco CA (2010) Review: Pathogen-induced inflammation at sites distant from oral infection: bacterial persistence and induction of cell-specific innate immune inflammatory pathways. Molecular oral microbiology 25: 305-316. DOI 10.1111/j.20411014.2010.00582.x

4. Hajishengallis G (2015) Periodontitis: from microbial immune subversion to systemic inflammation. Nature reviews Immunology 15: 30-44. DOI 10.1038/nri3785

5. Irie K, Novince CM, Darveau RP (2014) Impact of the Oral Commensal Flora on Alveolar Bone Homeostasis. Journal of dental research 93: 801-806. DOI 10.1177/0022034514540173

6. Wallach D, Kang TB, Kovalenko A (2014) Concepts of tissue injury and cell death in inflammation: a historical perspective. Nature reviews Immunology 14: 51-59. DOI 10.1038/nri3561

7. Newton K, Manning G (2016) Necroptosis and Inflammation. Annual review of biochemistry 85: 743763. DOI 10.1146/annurev-biochem-060815-014830

8. Pasparakis M, Vandenabeele P (2015) Necroptosis and its role in inflammation. Nature 517: 311320. DOI 10.1038/nature14191

9. Wallach D, Kang TB, Dillon CP, Green DR (2016) Programmed necrosis in inflammation: Toward identification of the effector molecules. Science 352: aaf2154. DOI 10.1126/science.aaf2154

10. Chan FK, Luz NF, Moriwaki K (2015) Programmed necrosis in the cross talk of cell death and inflammation. Annual review of immunology 33: 79-106. DOI 10.1146/annurev-immunol-032414112248

11. Shlomovitz I, Zargrian S, Gerlic M (2017) Mechanisms of RIPK3-induced inflammation. Immunology \& Cell Biology 95: 166-172. DOI 10.1038/icb.2016.124

12. Silke J, Rickard JA, Gerlic M (2015) The diverse role of RIP kinases in necroptosis and inflammation. Nature immunology 16: 689-697. DOI 10.1038/ni.3206

13. Song B, Zhou T, Yang WL, Liu J, Shao LQ (2017) Programmed cell death in periodontitis: recent advances and future perspectives. Oral diseases 23: 609-619. DOI 10.1111/odi.12574

14. Manosudprasit A, Kantarci A, Hasturk H, Stephens D, Van Dyke TE (2017) Spontaneous PMN apoptosis in type 2 diabetes and the impact of periodontitis. Journal of leukocyte biology 102: 14311440. DOI 10.1189/jlb.4A0416-209RR

15. Bullon P, Cordero MD, Quiles JL, Ramirez-Tortosa MDC, Gonzalez-Alonso A, Alfonsi S, García-Marín R, de Miguel M, Battino M (2012) Autophagy in periodontitis patients and gingival fibroblasts: unraveling the link between chronic diseases and inflammation. BMC medicine 10: 122-122. DOI $10.1186 / 1741-7015-10-122$ 
16. Linkermann A, Green DR (2014) Necroptosis. The New England journal of medicine 370: 455-465. DOI 10.1056/NEJMra1310050

17. Weinlich R, Oberst A, Beere HM, Green DR (2017) Necroptosis in development, inflammation and disease. Nat Rev Mol Cell Biol 18: 127-136. DOI 10.1038/nrm.2016.149

18. Vercammen D, Beyaert R, Denecker G, Goossens V, Van Loo G, Declercq W, Grooten J, Fiers W, Vandenabeele $P$ (1998) Inhibition of caspases increases the sensitivity of L929 cells to necrosis mediated by tumor necrosis factor. The Journal of experimental medicine 187: 1477-1485. DOI 10.1084/jem.187.9.1477

19. Challa S, Chan FK (2010) Going up in flames: necrotic cell injury and inflammatory diseases. Cellular and molecular life sciences : CMLS 67: 3241-3253. DOI 10.1007/s00018-010-0413-8

20. Oberst A, Green DR (2011) It cuts both ways: reconciling the dual roles of caspase 8 in cell death and survival. Nat Rev Mol Cell Biol 12: 757-763. DOI 10.1038/nrm3214

21. Kim SJ, Li J (2013) Caspase blockade induces RIP3-mediated programmed necrosis in Toll-like receptor-activated microglia. Cell Death Dis 4: e716. DOI 10.1038/cddis.2013.238

22. Kaiser WJ, Upton JW, Long AB, Livingston-Rosanoff D, Daley-Bauer LP, Hakem R, Caspary T, Mocarski ES (2011) RIP3 mediates the embryonic lethality of caspase-8-deficient mice. Nature 471: 368-372. DOI 10.1038/nature09857

23. Humphries F, Yang S, Wang B, Moynagh PN (2015) RIP kinases: key decision makers in cell death and innate immunity. Cell Death \& Differentiation 22: 225-236. DOI 10.1038/cdd.2014.126

24. Vanden Berghe T, Linkermann A, Jouan-Lanhouet S, Walczak H, Vandenabeele P (2014) Regulated necrosis: the expanding network of non-apoptotic cell death pathways. Nat Rev Mol Cell Biol 15: 135147. DOI 10.1038/nrm3737

25. Zelic M, Roderick JE, O'Donnell JA, Lehman J, Lim SE, Janardhan HP, Trivedi CM, Pasparakis M, Kelliher MA (2018) RIP kinase 1-dependent endothelial necroptosis underlies systemic inflammatory response syndrome. J Clin Invest 128: 2064-2075. DOI 10.1172/JCI96147

26. Jouan-Lanhouet S, Riquet F, Duprez L, Vanden Berghe T, Takahashi N, Vandenabeele P (2014) Necroptosis, in vivo detection in experimental disease models. Semin Cell Dev Biol 35: 2-13. DOI 10.1016/j.semcdb.2014.08.010

27. Shi J, Li J, Su W, Zhao S, Li H, Lei L (2019) Loss of periodontal ligament fibroblasts by RIPK3-MLKLmediated necroptosis in the progress of chronic periodontitis. Scientific reports 9: 2902. DOI 10.1038/s41598-019-39721-1

28. Ke X, Lei L, Li H, Li H, Yan F (2016) Manipulation of necroptosis by Porphyromonas gingivalis in periodontitis development. Mol Immunol 77: 8-13. DOI 10.1016/j.molimm.2016.07.010

29. Li J, Shi J, Pan Y, Zhao Y, Yan F, Li H, Lei L (2019) Transcription modulation by CDK9 regulates inflammatory genes and RIPK3-MLKL-mediated necroptosis in periodontitis progression. Scientific reports 9: 17369. DOI 10.1038/s41598-019-53910-y

30. Dutzan N, Abusleme L, Bridgeman H, Greenwell-Wild T, Zangerle-Murray T, Fife ME, Bouladoux N, Linley H, Brenchley L, Wemyss K, et al. (2017) On-going Mechanical Damage from Mastication 
Drives Homeostatic Th17 Cell Responses at the Oral Barrier. Immunity 46: 133-147. DOI 10.1016/j.immuni.2016.12.010

31. Ahmed FE, Gouda MM, Hussein LA, Ahmed NC, Vos PW, Mohammad MA (2017) Role of Melt Curve Analysis in Interpretation of Nutrigenomics' MicroRNA Expression Data. Cancer Genomics Proteomics 14: 469-481. DOI 10.21873/cgp.20057

32. Jouan-Lanhouet S, Riquet F, Duprez L, Vanden Berghe T, Takahashi N, Vandenabeele P (2014) Necroptosis, in vivo detection in experimental disease models. Seminars in Cell \& Developmental Biology 35: 2-13. DOI https://doi.org/10.1016/j.semcdb.2014.08.010

33. He S, Liang Y, Shao F, Wang X (2011) Toll-like receptors activate programmed necrosis in macrophages through a receptor-interacting kinase-3-mediated pathway. Proceedings of the National Academy of Sciences of the United States of America 108: 20054-20059. DOI 10.1073/pnas. 1116302108

34. Zenobia C, Hajishengallis G (2015) Porphyromonas gingivalis virulence factors involved in subversion of leukocytes and microbial dysbiosis. Virulence 6: 236-243. DOI 10.1080/21505594.2014.999567

35. Wang H, Sun L, Su L, Rizo J, Liu L, Wang LF, Wang FS, Wang X (2014) Mixed lineage kinase domainlike protein MLKL causes necrotic membrane disruption upon phosphorylation by RIP3. Mol Cell 54: 133-146. DOI 10.1016/j.molcel.2014.03.003

36. Kondylis V, Kumari S, Vlantis K, Pasparakis M (2017) The interplay of IKK, NF-KB and RIPK1 signaling in the regulation of cell death, tissue homeostasis and inflammation. Immunological reviews 277: 113-127. DOI 10.1111/imr.12550

\section{Table}

Table. Primers Sequence for qRT-PCR

\begin{tabular}{|l|l|l|}
\hline Gene & Forward $\left(5^{\prime}\right.$ to $3^{\prime}$ ) & Reverse $\left(5^{\prime}\right.$ to $3^{\prime}$ ) \\
\hline Human gapdh & TGTGTCCGTCGTGGATCTGA & CCTGCTTCACCACCTTCTTGA \\
\hline Human Ripk3 & CAGTGTGCAACAGGCAGAAC & CAGTGTGCAACAGGCAGAAC \\
\hline $\begin{array}{l}\text { Human } \\
\text { Glkl }\end{array}$ & TGAGGCAGTCTTTGGAATCGT & GGCTGAGCCAGTCTTCACATT \\
\hline Tnf- $\alpha$ & TGACCACAGTCCATGCCATC & GACGGACACATTGGGGGTAG \\
\hline$I l-6$ & CCCTCACACTCAGATCATCTTCT & GCTACGACGTGGGCTACAG \\
\hline Cox2 & TGTGCAATGGCAATTCTGAT & CTCTGAAGGACTCTGGCTTTG \\
\hline$M m p 9$ & CCCTGAAGCCGTACACATCA & TGTCACTGTAGAGGGCTTTCAATT \\
\hline Rankl & GCTGACTACGATAAGGACGGCA & GCGGCCCTCAAAGATGAACGG \\
\hline
\end{tabular}

\section{Figures}



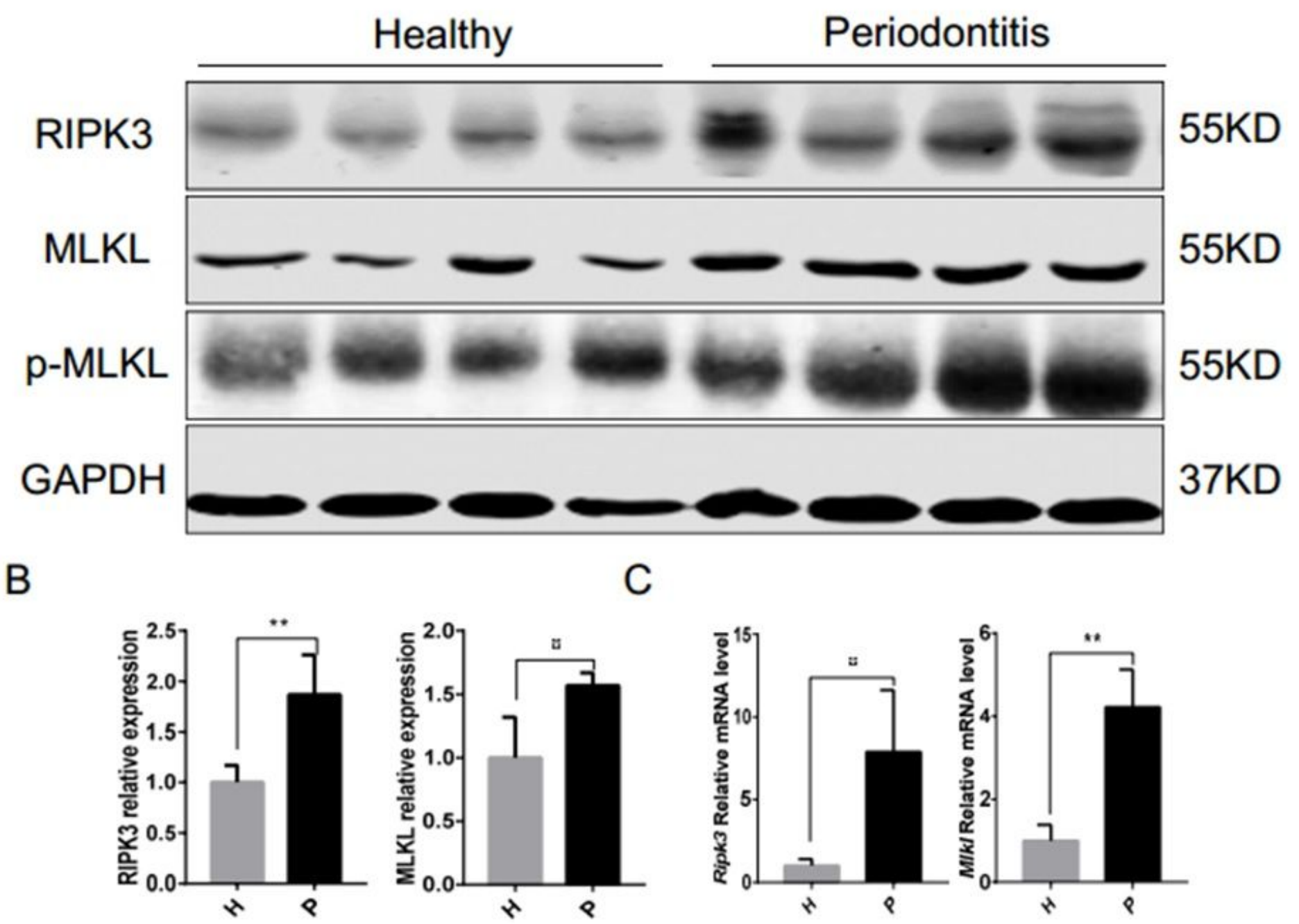

Figure 1

Necroptosis is involved in the pathogenesis of periodontitis. (A) Western blots of RIPK3, MLKL, and pMLKL proteins in gingival tissues from healthy subjects and periodontitis patients (both $n=4)$. (B) Relative protein levels after normalization to GAPDH. (C) Ripk3 and Mlkl mRNA levels normalized to human gapdh expression in gingival tissues from healthy subjects and periodontitis patients.

Abbreviations: $\mathrm{H}$, healthy subjects; $\mathrm{P}$, periodontitis patients; RIPK3, receptor interacting protein kinase-3; MLKL, mixed lineage kinase domain-like pseudokinase; $\mathrm{p}-\mathrm{MLKL}$, phosphorylated MLKL. 
A

\section{BMDMs}
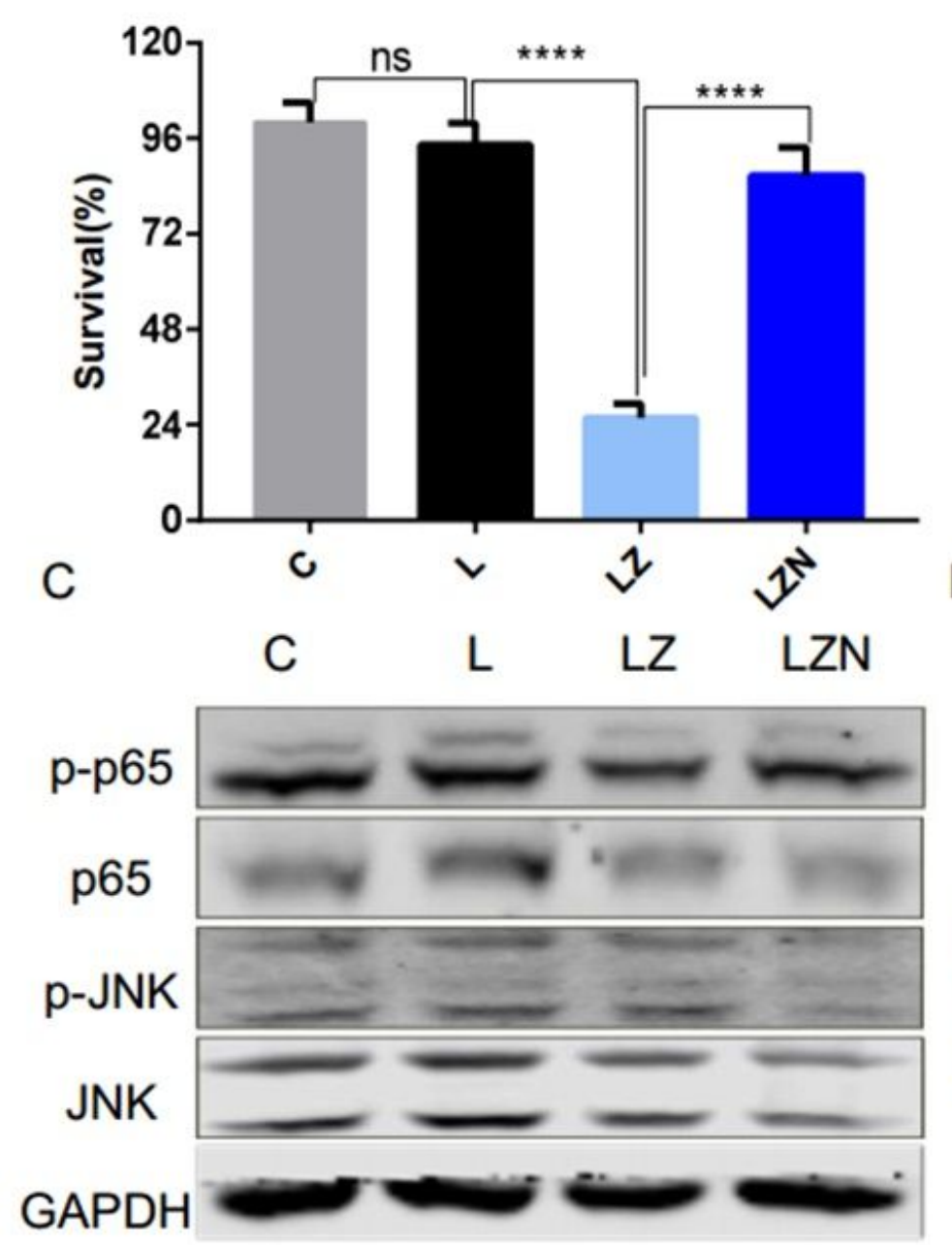

B
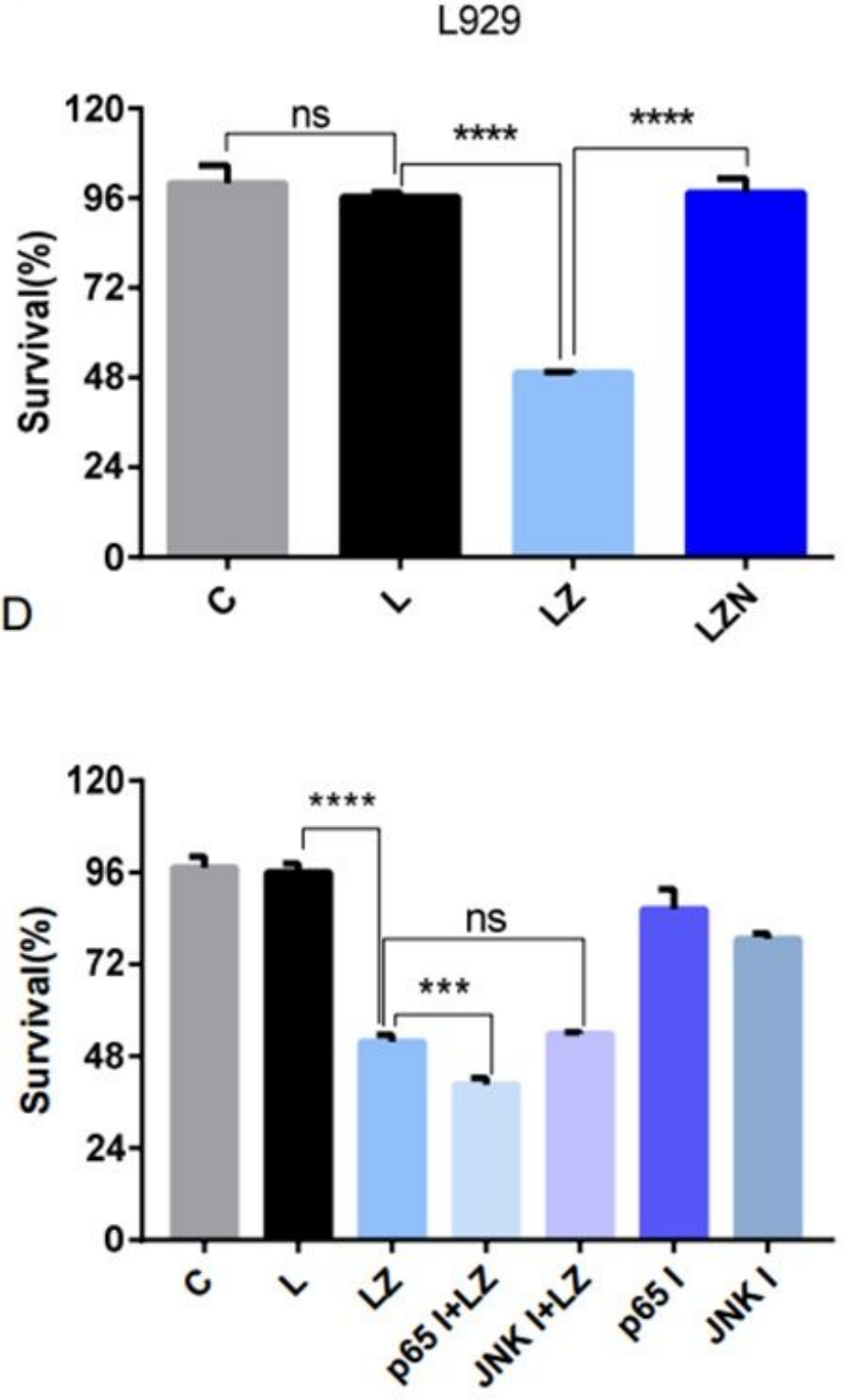

Figure 2

LPS-Pg induces necroptosis in a caspase-independent manner and negative regulates the NF-KB signaling pathway. (A) Viability of BMDM cells treated with $1 \mu \mathrm{g} / \mathrm{mL}$ LPS-Pg, zVAD $20 \mu \mathrm{M}$, or Nec-1 30 $\mu \mathrm{M}$ for $24 \mathrm{~h}$. (B) Viability of L929 cells treated with $1 \mu \mathrm{g} / \mathrm{mL}$ LPS-Pg, zVAD $20 \mu \mathrm{M}$, or Nec-130 $\mu \mathrm{M}$ for 24 h. (C) Western blots of p65, phosphorylated (p)-p65, JNK, and p-JNK proteins in L929 cells treated with LPS-Pg $1 \mu \mathrm{g} / \mathrm{mL}$, zVAD $20 \mu \mathrm{M}$, or Nec-1 $30 \mu \mathrm{M}$ for $24 \mathrm{~h}$. (D) Viability of L929 cells pretreated with a p65 or JNK inhibitor for $1 \mathrm{~h}$ and then treated with $1 \mu \mathrm{g} / \mathrm{mL}$ LPS-Pg and zVAD $20 \mu \mathrm{M}$ for $24 \mathrm{~h}$. Data representative of three separate experiments. Values are expressed as means \pm SEM. Abbreviations: LPS-Pg, Porphyromonas gingivalis lipopolysaccharide, Nec-1, necrostatin-1; JNK, c-jun N-terminal kinase; C, control; L, LPS-Pg; LZ, LPS-Pg + zVAD; LZN, LPS-Pg + zVAD + Nec-1; p65 I + LZ, p65 inhibitor + LPS-Pg + zVAD; JNK I + LZ, JNK inhibitor + LPS-Pg + zVAD; p65 I, p65 inhibitor; JNK I, JNK inhibitor. ns, statistically non-significant difference $(P>0.05)$; $* \star \star * P<0.0001 ; * \star * P<0.001$. 
A

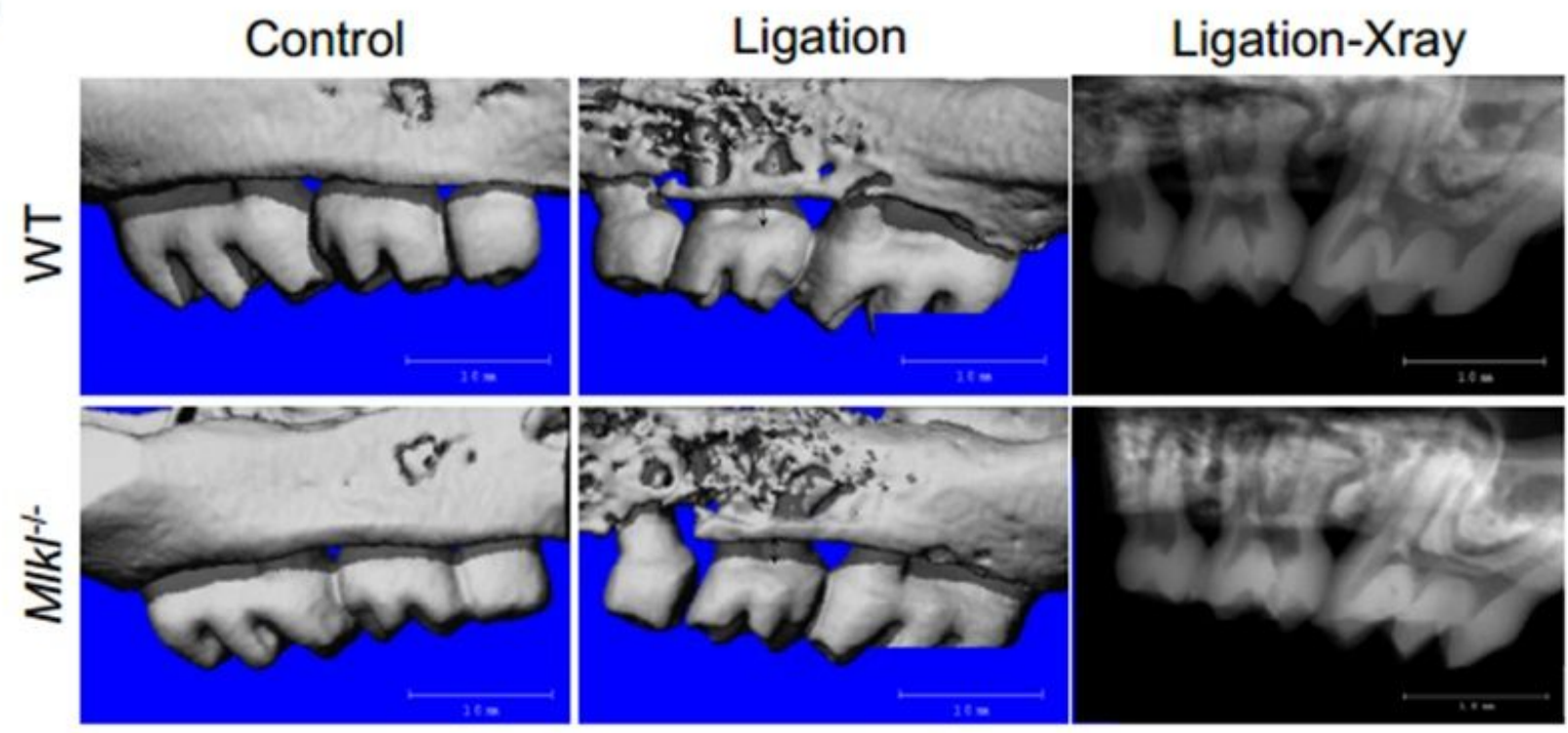

B

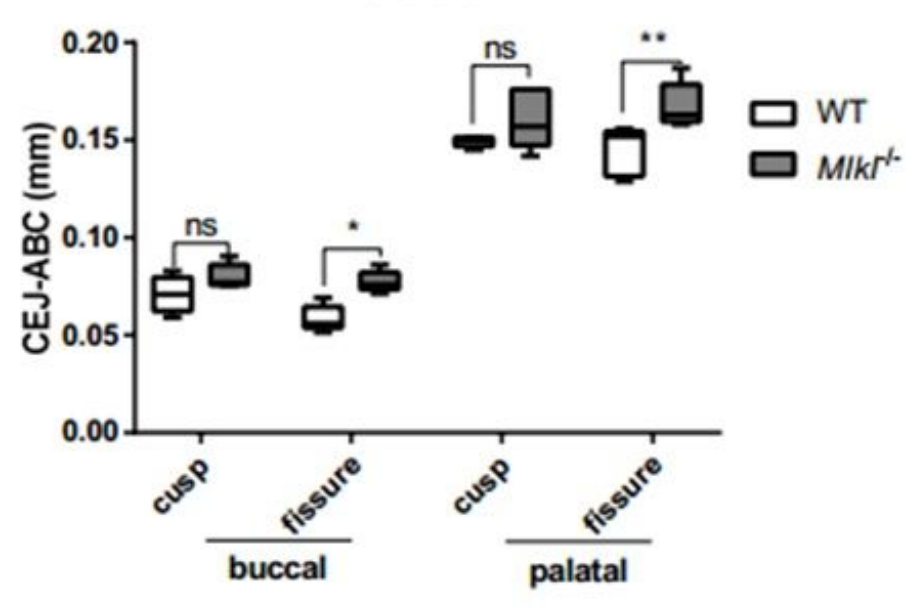

C

Buccal ABL

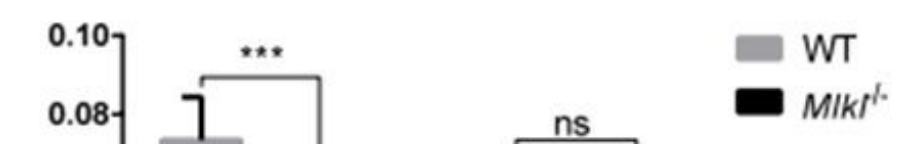

\section{Figure 3}

Mlkl deficiency protects against alveolar bone loss in mice with experimentally induced periodontitis. (A) Representative micro-computed tomography views of the maxillary molars from WT and Mlkl-/- mice after ligation. Arrows in the images correspond to the CEJ-ABC distances on the buccal side of the ligated teeth. Scale bars, $1 \mathrm{~mm}$. (B) Measurements of CEJ-ABC distances on the buccal and palatal sides of control teeth in WT and Mlkl-/- mice. (C) Measurements of alveolar bone loss on the buccal side of ligated teeth in WT and Mlkl-/- mice. Results are expressed as means \pm SEM. Abbreviations: ABC, alveolar bone crest; $\mathrm{ABL}$, alveolar bone loss; CEJ, cementoenamel junction; WT, wild-type; Mlkl, mixed lineage kinase domain-like pseudokinase. ns, statistically non-significant difference $(P>0.05)$; $* P<0.05$; $\star * \mathrm{P}<0.01$. 
A

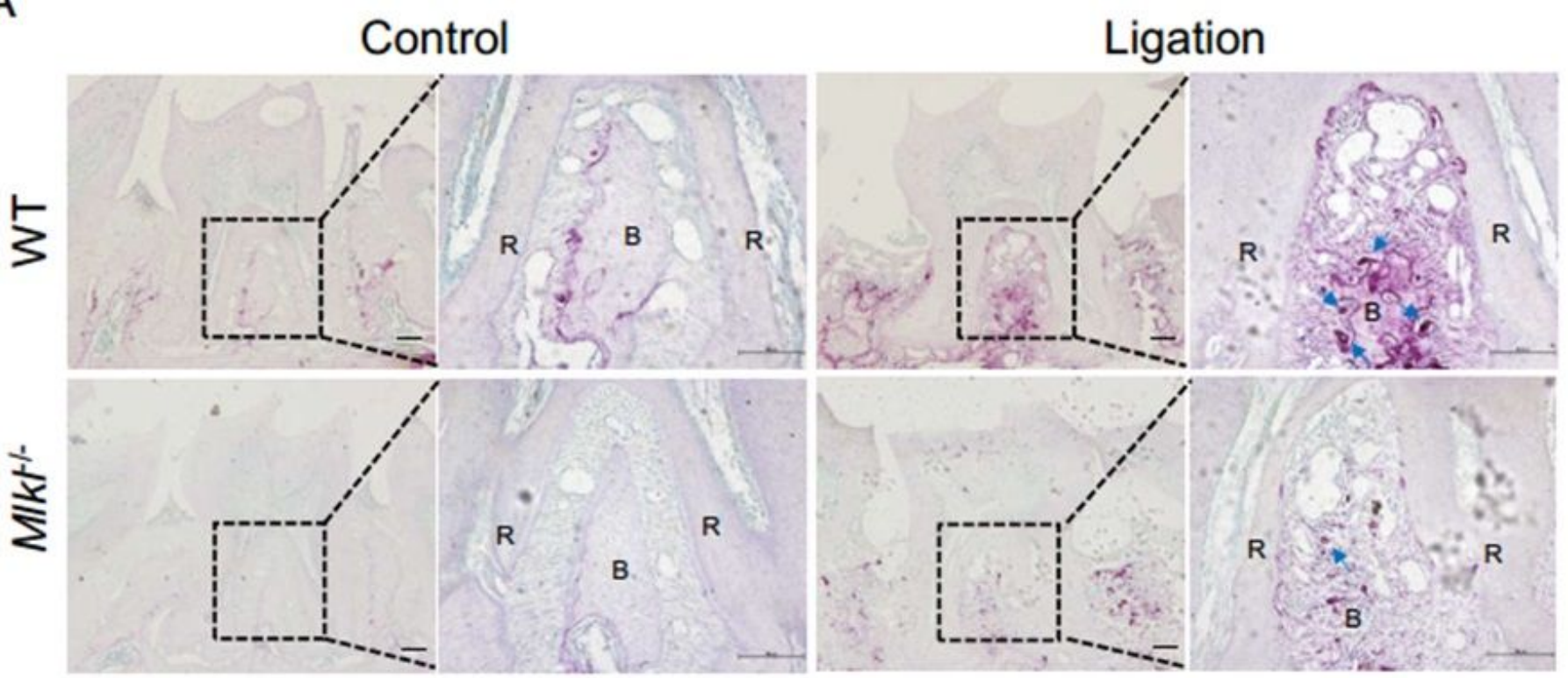

B

C

Control

Ligation

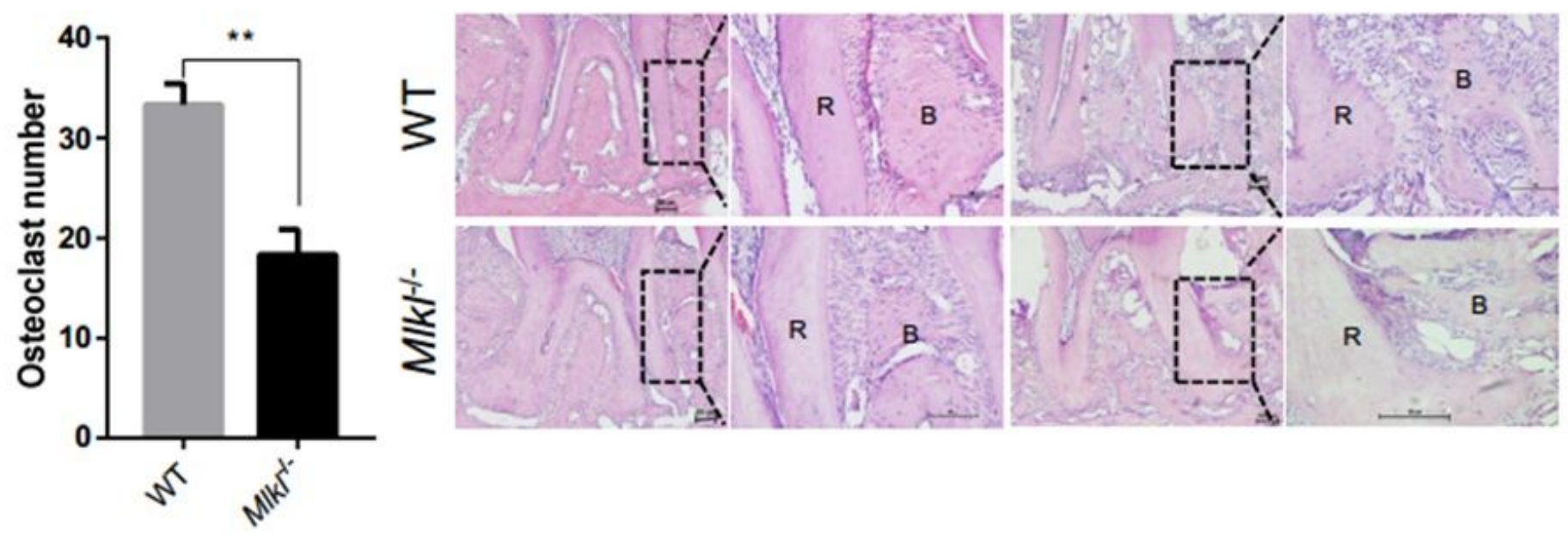

Figure 4

Mlkl-/- mice with experimentally induced periodontitis show a marked decrease in osteoclast activation. (A) Representative tartrate-resistant acid phosphatase-stained sections of teeth and periodontal tissues from WT and Mlkl-/- mice (left panels, 4× magnification, scale bar, $100 \mu \mathrm{m}$; right panels, 10x magnification, scale bar, $100 \mu \mathrm{m}$ ). Blue arrowheads indicate osteoclasts. (B) Total osteoclast number in ligated second molars. Results are expressed as means \pm SEM. (C) Representative hematoxylin and eosin-stained sections of periodontal tissues from WT and Mlkl-/- mice (left panels, $4 \times$ magnification, scale bar, $100 \mu \mathrm{m}$; right panels, 20× magnification, scale bar, $100 \mu \mathrm{m}$ ). Abbreviations: WT, wild-type; Mlkl, mixed lineage kinase domain-like pseudokinase; $R$, tooth root; $B$, alveolar bone. ${ }^{* \star} P<0.01$. 
A
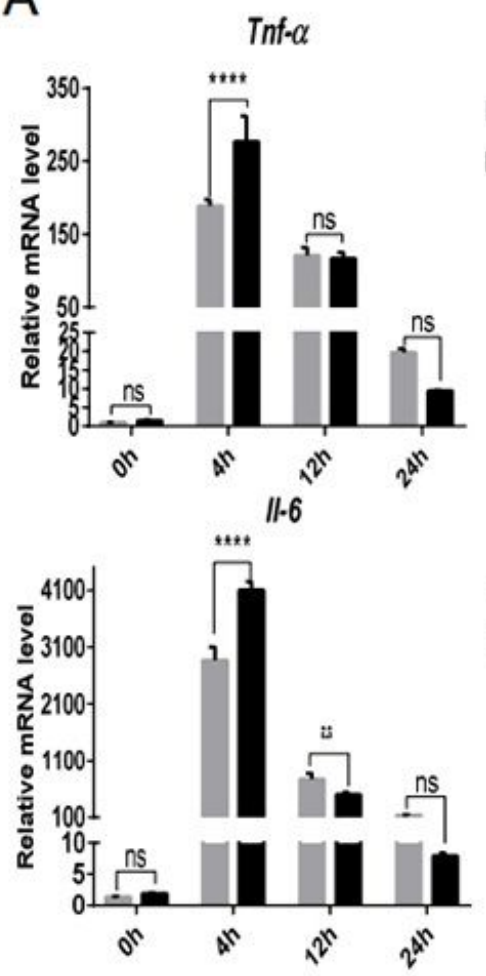

$\|-1 \beta$
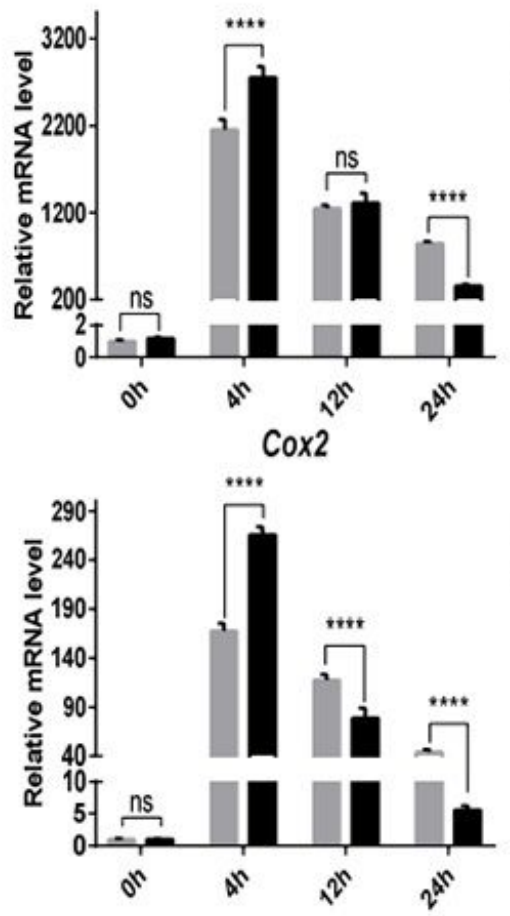

B

WT
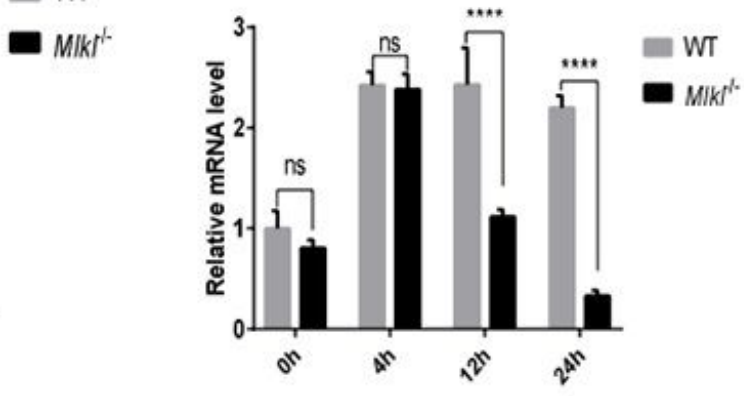

WT

Rankl

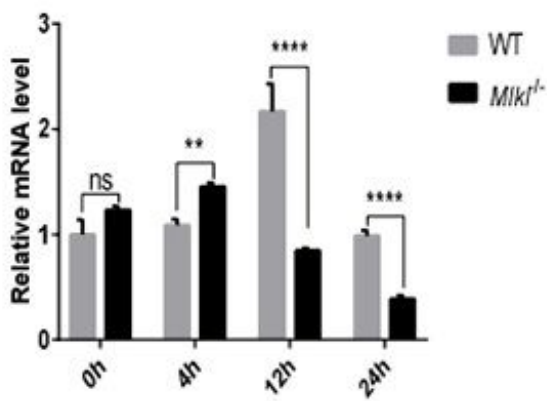

Figure 5

Time course of changes in the expression of inflammatory cytokine- and osteoclast-related genes in BMDMs of WT and Mlkl-/- mice. (A) mRNA levels of II-6, II-1 $\beta$, Tnf-a, and Cox2. (B) mRNA levels of Mmp9 and Rankl in BMDMs stimulated by LPS-Pg for $0,4,12$ and $24 \mathrm{~h}$. Values are presented as means \pm SEM. Abbreviations: BMDMs, bone marrow-derived macrophages; WT, wild-type; Mlkl, mixed lineage kinase domain-like pseudokinase; LPS-Pg, Porphyromonas gingivalis lipopolysaccharide; II, interleukin; Tnf-a, tumor necrosis factor-alpha; Cox2, cyclooxygenase 2 . ${ }^{*} \mathrm{P}<0.05$, ${ }^{\star *} \mathrm{P}<0.01$, ${ }^{\star \star \star \star} \mathrm{P}<0.0001$; ns, statistically non-significant difference $(P>0.05)$. 